\title{
Utilidad de la ecografía en la valoración de la extensión de la colitis ulcerosa
}

\author{
D. Martínez Ares, J. Martínez Cadilla, D. Rodríguez Martínez, S. Pereira Bueno, I. Martín-Granizo \\ Barrenechea, J. I. Rodríguez Prada, L. Cid Gómez y A. Pallarés Peral \\ Servicio de Aparato Digestivo. Complejo Hospitalario Xeral-Cies. Complejo Hospitalario Universitario de Vigo. \\ Pontevedra
}

\section{RESUMEN}

Introducción: el hallazgo de lesiones endoscópicas severas en un paciente con colitis ulcerosa desaconseja la realización de una exploración completa del colon. No obstante el conocimiento de la extensión precisa de la enfermedad tiene gran importancia en las decisiones terapéuticas a tomar y también en el pronóstico de la enfermedad. Por todo ello, la validación de una técnica no invasiva para el estudio de extensión de la colitis ulcerosa cobra gran interés e importancia.

Material y método: se incluyen en el estudio pacientes con diagnóstico previo de colitis ulcerosa o en el debut de la enfermedad y, de forma prospectiva y ciega se evalúa la precisión de la ecografía digestiva en la valoración de la extensión de la colitis ulcerosa. Las exploraciones ecográficas son realizadas todas ellas por el mismo explorador y siempre con anterioridad al estudio endoscópico completo, que se usa como patrón oro. No se emplea la técnica hidrocolónica en ningún caso.

Resultados: han sido incluidos en el estudio 20 pacientes, 13 varones (65\%) y 7 mujeres (35\%), con una edad media de 51,7 años (rango de 24 a 82 años). Los estudios endoscópicos mostraron una afectación severa en 5 casos (25\%), moderada en 12 pacientes $(60 \%)$ y lesiones leves en los 3 casos restantes (15\%). El estudio ecográfico del colon fue considerado satisfactorio en 18 casos $(90 \%)$ y la extensión de la enfermedad establecida en el estudio ecográfico coincide en todos los casos con la determinada por la colonoscopia: 3 pacientes $(16,6 \%)$ presentaban una proctitis ulcerosa, $9(50 \%)$ una colitis izquierda y $6(33,3 \%)$ una colitis extensa.

Conclusiones: la ecografía digestiva permite el estudio del marco colónico en la mayoría de los pacientes, especialmente si existe actividad inflamatoria, permitiendo establecer con gran precisión la extensión de la colitis ulcerosa, independientemente del grado de actividad de la misma.

Palabras clave: Colitis ulcerosa. Diagnóstico. Extensión. Ecografía. Endoscopia.

\begin{abstract}
Introduction: a full examination of the colon should be avoided upon finding severe endoscopic lesions in patients with ulcerative colitis. However, knowledge of the precise extent of disease is quite important for disease prognosis and the making of therapeutic decisions. Therefore, any validation of a non-invasive technique to assess the extent of ulcerative colitis gains a lot of interest and importance.

Material and method: the study included patients that were previously diagnosed of having ulcerative colitis or were beginning to suffer from the disease. A prospective and blind evaluation was carried out to determine the precision of digestive ultrasonography in assessment of ulcerative colitis extent. All ultrasonography was carried out by the same person and was always performed prior to carrying out a full endoscopic study, which is used as the gold standard. The hydrocolonic ultrasonograpy technique was not used in any of the cases.

Results: a total of 20 patients -13 males (65\%) and 7 females (35\%), with an average age of 51.7 years (aged between 24-82 years)- were included in the study. Endoscopic studies revealed severe disease in 5 cases (25\%), moderate disease in 12 patients (60\%), and mild lesions in the 3 remaining cases (15\%). A colonic ultrasonogram was considered satisfactory in 18 cases (90\%), and the extent of disease as established by ultrasonography was in all cases consistent with that established through colonoscopy: 3 patients (16.6\%) had ulcerative proctitis, 9 patients (50\%) had leftsided ulcerative colitis, and 6 (33.3\%) had extensive colitis.

Conclusions: digestive ultrasonography allows to study the colon in most patients, especially when inflammatory activity is present, and provides a greater accuracy in assessing ulcerative colitis extent, which is independent of its activity level.
\end{abstract}

Key words: Ulcerative colitis. Diagnosis. Extent. Ultrasonography. Endoscopy.

Martínez Ares D, Martínez Cadilla J, Rodríguez Martínez D, Pereira Bueno S, Martín-Granizo Barrenechea I, Rodríguez Prada JI, Cid Gómez L, Pallarés Peral A. Utilidad de la ecografía digestiva en la valoración de la extensión de la colitis ulcerosa. Rev Esp Enferm

Recibido: 15-12-06

Aceptado: 27-02-07.

Correspondencia: David Martínez Ares. Servicio de Aparato Digestivo. Hospital Xeral-Cíes. C/ Pizarro, 22. 36204 Vigo, Pontevedra. e-mail: david.martinez.ares@sergas.es 


\section{INTRODUCCIÓN}

El hallazgo de lesiones endoscópicas graves durante la realización de una colonoscopia en un paciente con colitis ulcerosa desaconseja la realización de una exploración completa del colon por el elevado riesgo de complicaciones importantes, principalmente megacolon tóxico o perforación (1). Por otra parte, ante una recidiva clínica de una enfermedad conocida puede resultar de interés la evaluación de la extensión de la enfermedad para descartar una progresión de la misma. Ello exige, en principio, la realización de una colonoscopia, una técnica invasiva y no siempre bien aceptada por el paciente.

El conocimiento de la extensión de la enfermedad así como de la gravedad de la afectación puede conllevar modificaciones en la actitud terapéutica, ya que en casos de afectación distal puede ser suficiente un tratamiento tópico, evitando así fármacos sistémicos, algunos de ellos con importantes efectos secundarios (2-5). Adicionalmente, el conocimiento de la extensión de la colitis ulcerosa es importante porque marca diferencias pronósticas: mayor riesgo de precisar ingreso hospitalario y colectomía, de degeneración en cáncer de colon y de mortalidad en las formas más extensas (6-10).

La extensión de la enfermedad no es inamovible, pudiendo objetivarse tanto la progresión de la enfermedad en las formas menos extensas como la regresión de la misma en las colitis extensas (11-13).

Clásicamente se ha clasificado la extensión de la colitis ulcerosa como proctitis cuando únicamente existe afectación distal a la unión rectosigmoidea, proctosigmoiditis cuando se afecta también el sigma, colitis izquierda si la afectación es más extensa pero sin sobrepasar el ángulo esplénico, colitis extensa si existe afectación proximal al ángulo esplénico y pancolitis si existe afectación proximal al ángulo hepático. No obstante, se ha comprobado que el pronóstico de proctosigmoiditis y colitis izquierda es muy parecido, de la misma forma que en la colitis extensa y pancolitis. De este modo, en la clasificación consensuada en Montreal en 2005 se diferencian únicamente tres grupos de pacientes según la extensión de su enfermedad: proctitis, colitis izquierda y colitis extensa (14).

Como ya se ha reseñado, el patrón oro en la evaluación de la extensión de la colitis ulcerosa es la colonoscopia, una técnica que exige preparación del intestino, suele requerir sedación del paciente para la realización de un examen completo, puede no ser bien tolerada por los pacientes y presenta un elevado riesgo de complicaciones en los pacientes con enfermedad activa severa (1). Por este motivo, se ha evaluado la utilidad de diversas técnicas radiológicas y la gammagrafía con leucocitos marcados como técnicas menos invasivas en el estudio de extensión de la enfermedad con resultados dispares. Nosotros evaluamos en el siguiente estudio la precisión de la ecografía abdominal, una técnica fácilmente accesible, prácticamente inocua, bien tolerada por el paciente y que se puede realizar incluso "a pie de cama" en enfermos ingresados.

\section{MATERIAL Y MÉTODO}

\section{Selección de pacientes}

Han sido seleccionados pacientes con un diagnóstico previo de colitis ulcerosa o que presentan el debut de la misma en el momento actual, siendo incluidos únicamente aquellos en los que existe una confirmación histológica del diagnóstico. Han sido considerados tanto los pacientes en seguimiento ambulatorio que presentan una enfermedad activa como los pacientes con un primer brote de la enfermedad y con estudio endoscópico incompleto por los riesgos inherentes al mismo, en el caso de enfermedad grave, o por otros motivos (dificultad técnica o mala preparación del intestino) en los casos menos graves.

Han sido excluidos del análisis aquellos pacientes con enfermedad conocida sometidos a una exploración endoscópica del colon en los doce meses previos, ya que hemos considerado que el riesgo de progresión en la enfermedad en este periodo de tiempo podría ser lo suficientemente bajo como para condicionar los resultados del estudio ecográfico. Asimismo, no han sido incluidos aquellos pacientes en los que no se pudo completar un estudio endoscópico del colon, ya que esta técnica fue considerada como el patrón oro en el diagnóstico de extensión de la colitis ulcerosa. Finalmente, han sido excluidos del estudio aquellos pacientes en los que el diagnóstico de colitis ulcerosa no fuese concluyente en el examen histológico.

A todos los pacientes, dado que la ecografía no era la exploración para la que habían sido remitidos inicialmente en la mayoría de los casos, se les solicitó su consentimiento para la realización de la ecografía y para disponer de sus datos clínicos y analíticos para la realización del estudio.

\section{Metodología del estudio}

En todos los casos el estudio ecográfico del colon se realizó con anterioridad a la exploración endoscópica que permitió establecer de forma definitiva la extensión de la enfermedad. De este modo se asegura que el ecografista permanece "ciego" a los resultados de la colonoscopia. La técnica empleada en todos los casos fue la ecografía abdominal percutánea convencional, sin hacer uso de técnica hidrocolónica.

El examen ecográfico, en el caso de los pacientes ambulantes, se realizó como norma general el mismo día que la exploración endoscópica, por lo que se había realizado previamente una preparación intestinal con una solución polihidroelectrolítica convencional. En el caso de los pacientes ingresados la ecografía se llevó a cabo sin 
preparación alguna. Ambas exploraciones fueron realizadas durante el mismo ingreso, pero en ningún caso en el mismo día. Todos los estudios ecográficos fueron realizados por el mismo explorador, utilizando un ecógrafo Toshiba Nemio 10, dotado de una sonda convexa de $3,75 \mathrm{Mz}$ y una sonda lineal de 7,5 $\mathrm{MHz}$.

El estudio ecográfico del colon se inicia tras localizar la unión rectosigmoidea, y a partir de ahí se trata de seguir toda la extensión del colon hasta alcanzar la fosa ilíaca derecha, en la zona teórica del ciego. Se considera que el estudio ecográfico descarta actividad inflamatoria cuando se identifica una pared colónica que conserva su estructura en capas y con un grosor inferior a $4 \mathrm{~mm}$ (Figs. 1 y 2). Por el contrario, se considera el estudio patológico (Figs. 3 y 4) cuando el grosor de la pared supera los $4 \mathrm{~mm}$ acompañado o no de la alteración de la estructura en capas (no es habitual en la colitis ulcerosa, donde la inflamación se circunscribe generalmente a mucosa y submucosa). Dado que en la colitis ulcerosa la afectación mucosa suele ser continua, tras encontrar un segmento "normal", se puede descartar con bastante seguridad la existencia de segmentos patológicos situados proximalmente. Por ello, la ecografía se ha considerado satisfactoria cuando se logra identificar al menos un segmento colónico no afecto situado en contigüidad al segmento más proximal con características ecográficas patológicas. En el caso de los pacientes en los que únicamente hay afectación rectal, esta se supone, ya que la afectación de este ocurre en prácticamente todos los pacientes con colitis ulcerosa y en el estudio se han incluido sólo pacientes con confirmación histológica del diagnóstico, y asumimos el diagnóstico de proctitis cuando a partir de la unión rectosigmoidea y en sentido proximal el aspecto ecográfico de la pared del colon es normal.

\section{Variables estudiadas y estudio estadístico}

Se realiza una estadística descriptiva de todas las variables demográficas, clínicas y analíticas de los pacientes, con especial atención a los índices de gravedad y la extensión de la enfermedad desde el punto de vista endoscópico. Los criterios de clasificación utilizados corresponden a los términos que se establecen en la clasificación del consenso de Montreal 2005 (14). Se valora también el diagnóstico de extensión ecográfico y el grado de coincidencia entre los diagnósticos endoscópicos y ecográficos. Esta comparación se realiza mediante los coeficientes de contingencia que relacionan ambas variables. El estudio estadístico se llevó a cabo mediante el paquete estadístico SPSS 12.0 para Windows.

\section{RESULTADOS}

Han sido incluidos en el estudio 20 pacientes, 13 varones $(65 \%)$ y 7 mujeres $(35 \%)$, con una edad media de

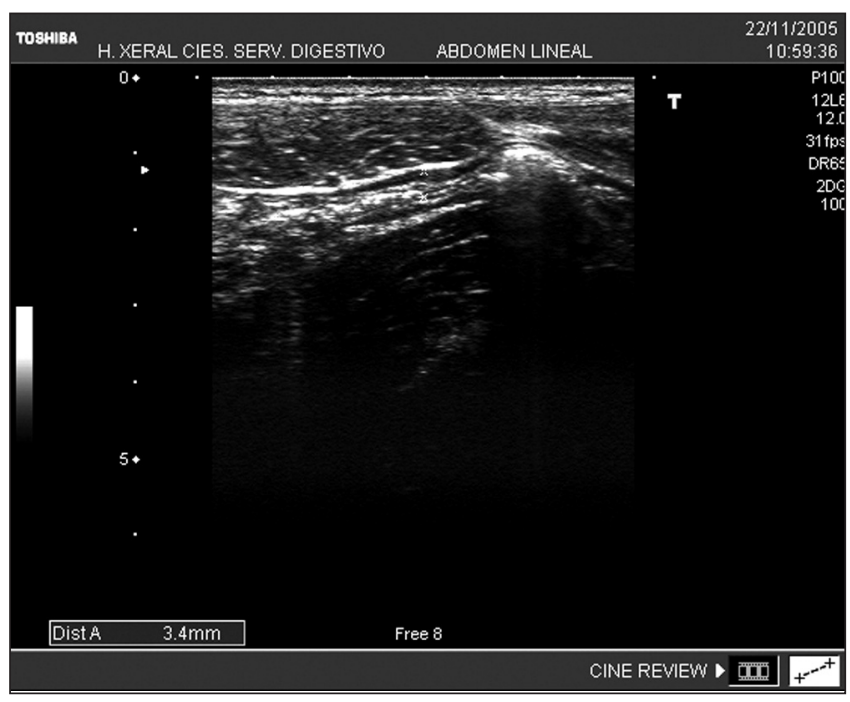

Fig. 1. Imagen obtenida con sonda lineal de alta frecuencia, a nivel de fosa ilíaca izquierda. Se observa un sigma normal, tras realizar una compresión gradual con el transductor a este nivel, con un grosor parietal de 3,4 mm y visualizándose con claridad las cinco capas ultrasonográficas habituales de la pared gastrointestinal, que se objetivan de forma simétrica a ambos lados de la imagen hiperecogénica creada por el gas existente en la luz intestinal.

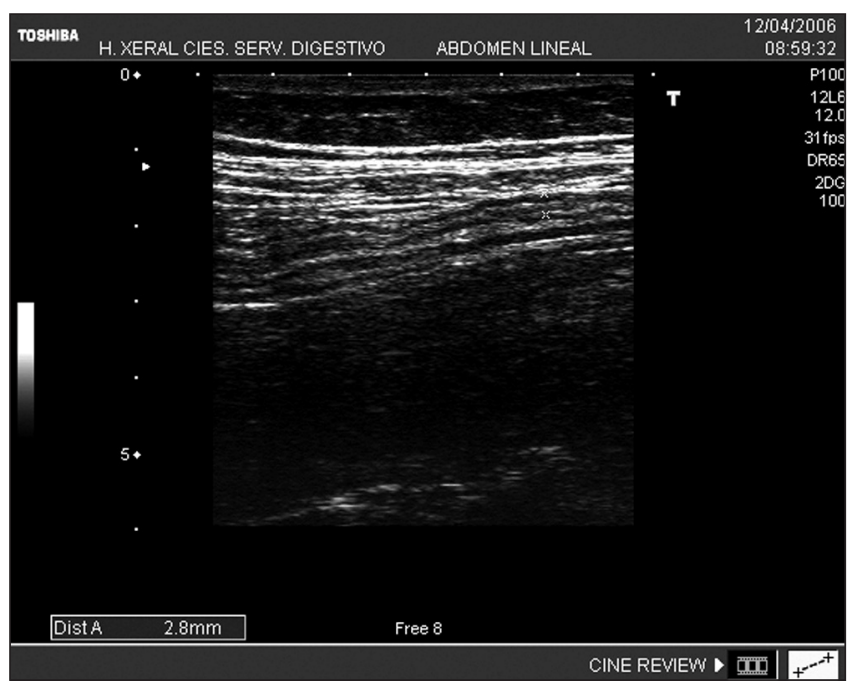

Fig. 2. Imagen obtenida con sonda lineal de alta frecuencia, a nivel de flanco izquierdo, estableciéndose de la misma forma que en el caso anterior, por debajo de los planos musculares un colon descendente con una ecoestructura normal y un grosor parietal de $2,8 \mathrm{~mm}$.

51,7 años (rango de 24 a 82 años). En 7 pacientes (35\%) la exploración ecográfica fue realizada tras un estudio endoscópico incompleto en el debut de la enfermedad (5 pacientes con actividad endoscópica severa y 2 pacientes con exploraciones incompletas como consecuencia de una preparación deficiente del colon). Por otra parte, 13 pacientes $(65 \%)$ presentaban recidivas clínicas de una enfermedad conocida y fueron remitidos desde la consulta ambulatoria para descartar una progresión en la ex- 


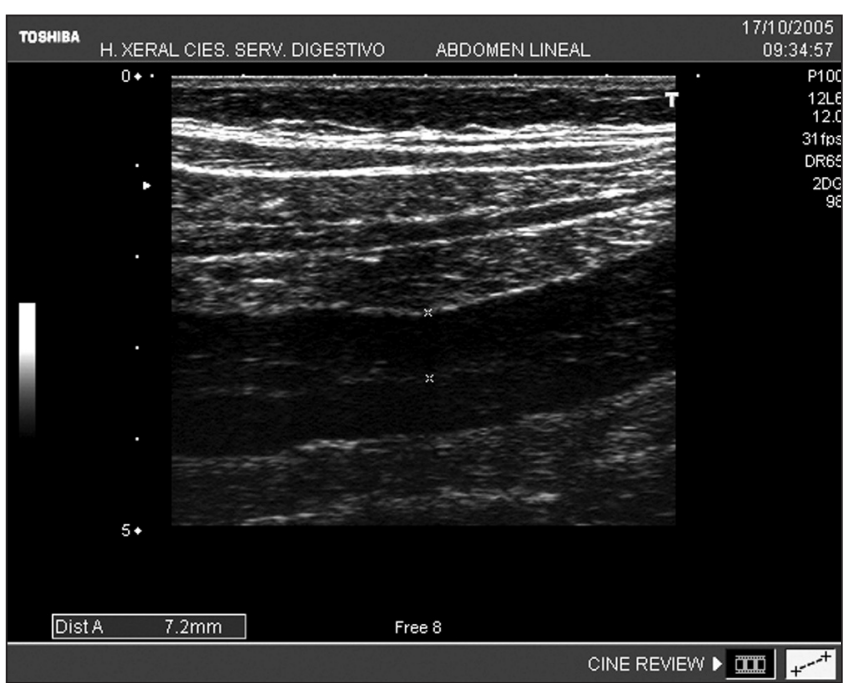

Fig. 3. Imagen obtenida con sonda lineal de alta frecuencia, a nivel de flanco izquierdo, observándose un engrosamiento de la pared del colon, que alcanza 7,2 mm, con una estructura en capas muy poco visible, aunque con unos límites a nivel de la serosa muy bien definidos y con un meso indemne. Los hallazgos corresponden a una colitis izquierda, moderada desde el punto de vista endoscópico.

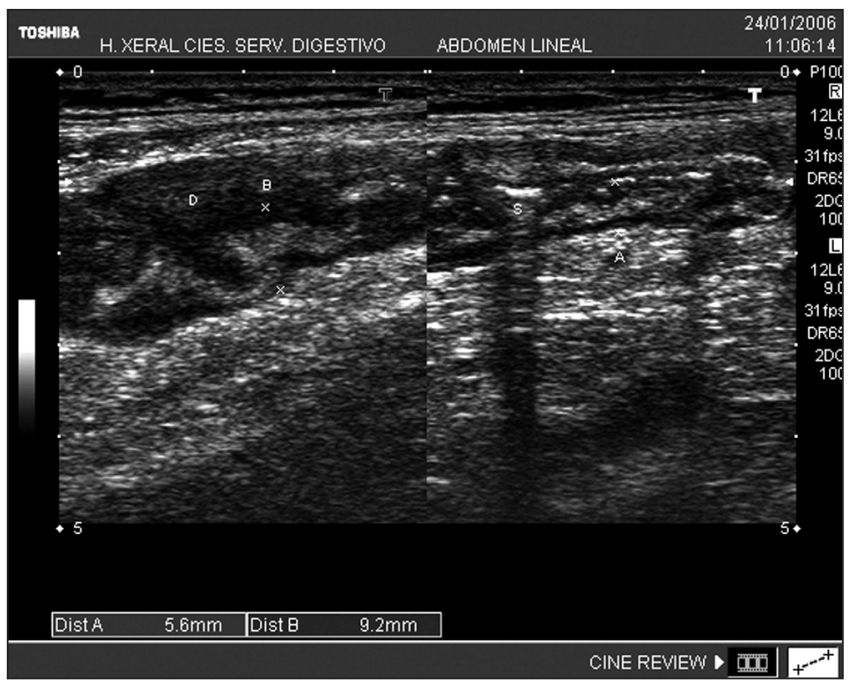

Fig. 4. Imágenes obtenidas con sonda lineal de alta frecuencia, a nivel de flanco izquierdo (derecha) y fosa iliaca izquierda (izquierda), objetivándose una pared del sigma y colon descendente engrosada, aunque con una estructura en capas conservada. La afectación es más intensa a nivel de mucosa y submucosa ( $2^{a}$ y $3^{a}$ capas), especialmente a nivel del sigma, donde se alcanza un espesor parietal de 9,2 mm. Los hallazgos corresponden a colitis izquierda moderada-severa desde el punto de vista endoscópico.

tensión de la colitis. Los estudios endoscópicos mostraron una afectación severa en 5 casos $(25 \%)$, moderada en 12 pacientes $(60 \%)$ y lesiones leves en los 3 casos restantes $(15 \%)$ (Tabla I). El estudio ecográfico del colon fue considerado satisfactorio en 18 casos $(90 \%)$ y en 2 casos (10\%) las imágenes obtenidas no permitieron asegurar un diagnóstico de extensión. Uno de estos dos pacientes presentaba una afectación exclusivamente rectal (no se pudo visualizar un colon no afecto) y el otro paciente presentaba una colitis izquierda leve. En los 18 pacientes en los que se obtuvieron imágenes satisfactorias del colon, la extensión de la enfermedad establecida en el estudio ecográfico coincide en todos los casos con la determinada por la colonoscopia: 3 pacientes $(16,6 \%)$ presentaban una proctitis ulcerosa, $9(50 \%)$ una colitis izquierda y $6(33,3 \%)$ una colitis extensa. Todos estos datos se resumen también en la tabla I.

De estos datos se deduce en 18 de los 20 pacientes el diagnóstico de extensión de la colitis ulcerosa fue establecido de forma exacta por la ecografía abdominal, por lo que la precisión de la técnica en esta tarea alcanza el $90 \%$, y el coeficiente de contingencia que relaciona los diagnósticos endoscópicos y ecográficos es de 0,898 (p = 0,0018 ). Adicionalmente, sólo 2 pacientes no fueron estudiados correctamente y en sólo 1 caso (5\%) se tomarían decisiones terapéuticas inapropiadas en base a los hallazgos ecográficos (en una colitis izquierda podría no ser suficiente un tratamiento tópico).

\section{DISCUSIÓN}

El hallazgo de lesiones endoscópicas severas en un paciente con colitis ulcerosa contraindica la realización de un examen completo del colon, por el elevado riesgo de complicaciones graves como perforación o megacolon tóxico (1). Aunque esta no es una opinión aceptada por todos los autores $(15,16)$, es la práctica habitual en nuestro medio. No obstante, en estos brotes graves de activi-

Tabla I. Sumario de las indicaciones y resultados de los estudios endoscópicos y ecográficos a los que fueron sometidos a los pacientes incluidos en el estudio

\begin{tabular}{lcc}
\hline Variables & $\begin{array}{c}\text { Número } \\
\text { pacientes }\end{array}$ & \% pacientes \\
\hline Sexo & $13 / 20$ & \\
Varones & $7 / 20$ & 65 \\
Mujeres & & 35 \\
Indicación del estudio & \\
Debut de la enfermedad & $7 / 20$ & 35 \\
Descartar progresión & $13 / 20$ & 65 \\
Lesiones endoscópicas & & \\
Leves & $3 / 20$ & 15 \\
Moderadas & $12 / 20$ & 60 \\
Severas & $5 / 20$ & 25 \\
Extensión de la enfermedad en estudio endoscópico & \\
Proctitis & $4 / 20$ & 20 \\
Colitis izquierda & $10 / 20$ & 50 \\
Colitis extensa & $6 / 20$ & 30 \\
Extensión de la enfermedad en estudio ecográfico & \\
Proctitis & $3 / 18$ & 16,6 \\
Colitis izquierda & $9 / 18$ & 50 \\
Colitis extensa \\
Resultados de la valoración ecográfica de la extensión \\
Valoración correcta \\
Valoración inadecuada \\
\hline
\end{tabular}


dad, y especialmente en los casos en los que nos enfrentamos al debut de la enfermedad, el conocimiento exacto de la extensión de la enfermedad puede condicionar en gran medida las decisiones terapéuticas: cirugía, inmunosupresores, leucocitoaféresis, etc. (14). También en los pacientes con enfermedad menos severa y manejados ambulatoriamente podría tener cierto interés el poder descartar una progresión de la enfermedad mediante técnicas no invasivas, ya que muchos pacientes se muestran reticentes a someterse a exploraciones endoscópicas repetidas.

Han sido evaluadas múltiples técnicas radiológicas como la TAC abdominal, el enema baritado o la resonancia magnética, y también la ecografía como técnicas útiles en el diagnóstico diferencial de la enfermedad de Crohn y la pancolitis ulcerosa, así como en el diagnóstico de extensión de esta última, con resultados prometedores (1721). También se han evaluado técnicas isotópicas, como la resonancia magnética con gadolinio, la gammagrafía con leucocitos marcados o la tomografía de emisión de positrones (PET) (22-25). Sin embargo, algunas de estas técnicas de imagen requieren una preparación del intestino, que puede resultar incómoda y peligrosa, y otras no siempre están disponibles en la mayoría de los hospitales, como es el caso de la gammagrafía o la PET. De entre todas ellas, si hay alguna que destaca sobre todas las demás por su fácil acceso, inocuidad, escaso coste y la posibilidad de repetirla tantas veces como sea preciso, incluso "a pie de cama" en los enfermos en peores condiciones, es sin duda alguna la ecografía. No obstante, y al contrario de lo que ocurre en la enfermedad de Crohn, la rentabilidad de la ecografía digestiva en la valoración de los pacientes con colitis ulcerosa ha sido poco estudiada. En la mayoría de las series publicadas se incluyen pacientes con enfermedad de Crohn (la mayoría), y algunos con colitis ulcerosa. Por otra parte, en casi ninguno de ellos se hace uso de la ecografía para evaluar la extensión de la enfermedad. En cualquier caso, en estos estudios se establece que la ecografía se muestra muy sensible y específica en la detección de las lesiones de colitis ulcerosa. Las cifras de precisión global se encuentran entre el 63 y el $95 \%$ (21,26-33). Esta gran variabilidad en los resultados se explica en los diferentes patrones oro empleados en estos estudios para evaluar la precisión de la ecografía (endoscopia o técnicas radiológicas), la heterogeneidad de los pacientes seleccionados y las notables diferencias en los tamaños muestrales. Pascu y cols. (34) han publicado recientemente un estudio en el que incluyen 24 pacientes con colitis ulcerosa y donde la precisión mostrada por la ecografía para detectar lesiones de colitis ulcerosa en cualquier segmento del colon es del 95\% (91-100\%, en los diferentes segmentos), superando claramente a la resonancia, que alcanza una precisión del $81 \%$. La mayor limitación de la ecografía es que no permite estudiar la ampolla rectal. Nosotros no hemos evaluado la sensibilidad de la ecografía en el diagnóstico de la colitis ulcerosa, ya que partimos de pacientes con una enfermedad co- nocida; nuestro objetivo era la determinación de la extensión de la colitis, desconocida antes de la realización de la ecografía. El diagnóstico de extensión se basa, como hemos referido, en la detección de un segmento sano adyacente al segmento más proximal afecto. De este modo, aunque no podemos estudiar la ampolla rectal, en un paciente con afectación rectal conocida establecemos el diagnóstico de proctitis al visualizar un sigma normal desde el punto de vista ecográfico. Como ya se ha presentado, la precisión de la ecografía ha sido del $90 \%$ en la determinación de la extensión de la enfermedad. En los dos pacientes en los que el estudio no fue exitoso, curiosamente se trataba de pacientes ambulatorios que fueron estudiados con un colon preparado para colonoscopia. No nos parece probable que la ausencia de heces en el colon sea el motivo de que el estudio del colon no haya sido óptimo. En cualquier caso, el número reducido de pacientes no nos permite extraer conclusiones acerca de la conveniencia de realizar el estudio con o sin preparación del colon. No obstante, sólo en uno de los pacientes que fue incorrectamente diagnosticado se podrían llevar a cabo decisiones terapéuticas erróneas en base al estudio ecográfico. En cualquier caso, se trataba de un paciente con una colitis izquierda leve, y en algunos estudios se ha mostrado como el tratamiento tópico en forma de enemas (sólo de esta forma se consigue alcanzar el ángulo esplénico) podría ser tan eficaz como el tratamiento oral (35). En ningún caso de los pacientes que mostraban una enfermedad severa se erró en el diagnóstico, y consideramos que este es el grupo de pacientes que mayor beneficio obtiene de una prueba no invasiva para la valoración de la extensión de la enfermedad y tomar las decisiones terapéuticas pertinentes.

En conclusión, consideramos que hemos mostrado cómo la ecografía puede ser una herramienta útil para conocer la extensión de la colitis ulcerosa especialmente en aquellos pacientes en los que la práctica de una colonoscopia completa parece poco aconsejable. No obstante este se trata de un estudio preliminar, con pocos pacientes cuyos resultados han de ser confirmados en estudios más amplios.

\section{BIBLIOGRAFÍA}

1. Vázquez Iglesias JL. Enfermedad inflamatoria intestinal. En: Vázquez Iglesias JL, editor. Colonoscopia diagnóstica y terapéutica. A Coruña; 1998. p. 257-310.

2. Cohen RD, Woseth DM, Thisted RA, Hanauer SB. A meta-analysis and overview of the literature on treatment options for left-sided ulcerative colitis and ulcerative proctitis. Am J Gastroenterol 2000; 95: 1263-76.

3. Regueiro MD. Diagnosis and treatment of ulcerative proctitis. J Clin Gastroenterol 2004; 38: 733-40.

4. Marshall JK, Irvine EJ. Putting rectal 5-aminosalicylic acid in its place: The role in distal ulcerative colitis. Am J Gastroenterol 2000; 95 : 1628-36.

5. Marshall JK, Irvine EJ. Rectal corticosteroids versus alternative treatments in ulcerative colitis: A meta-analysis. Gut 1997; 40: 775-81.

6. Moum B, Ekbom A, Vatn MH, Aadland E, Sauar J, Lygren I, et al. 
Clinical course during the $1^{\text {st }}$ year after diagnosis in ulcerative colitis and Crohn's disease. Results of a large, prospective population-based study in southeastern Norway, 1990-93. Scand J Gastroenterol 1997; 32: 1005-12.

7. Farmer RG, Easley KA, Rankin GB. Clinical patterns, natural history, and progression of ulcerative colitis. A long-term follow-up of 1116 patients. Dig Dis Sci. 1993; 38: 1137-46.

8. Langholz E, Munkholm P, Davidsen M, Binder V. Colorectal cancer risk and mortality in patients with ulcerative colitis. Gastroenterology 1992; 103 (5): 1444-51.

9. Stewenius J, Adnerhill I, Anderson H, Ekelund GR, Floren CH, Fork $\mathrm{FT}$, et al. Incidence of colorectal cancer and all cause mortality in non-selected patients with ulcerative colitis and indeterminate colitis in Malmo, Sweden. Int J Colorectal Dis 1995; 10: 117-22.

10. Winther KV, Jess T, Langholz E, Munkholm P, Binder V. Survival and cause-specific mortality in ulcerative colitis: Follow-up of a population-based cohort in Copenhagen County. Gastroenterology 2003; 125: 1576-82.

11. Langholz E, Munkholm P, Davidsen M, Nielsen OH, Binder V. Changes in extent of ulcerative colitis: A study on the course and prognostic factors. Scand J Gastroenterol 1996; 31: 260-6.

12. Ayres RC, Gillen CD, Walmsley RS, Allan RN. Progression of ulcerative proctosigmoiditis: Incidence and factors influencing progression. Eur J Gastroenterol Hepatol 1996; 8: 555-8.

13. Meucci G, Vecchi M, Astegiano M, Beretta L, Cesari P, Dizioli P, et al. The natural history of ulcerative proctitis: A multicenter, retrospective study. Gruppo di Studio per le Malattie Infiammatorie Intestinali (GSMII). Am J Gastroenterol 2000; 95: 469-73.

14. Silverberg MS, Satsangi J, Ahmad T, Arnott ID, Bernstein CN, Brant $\mathrm{SR}$, et al. Toward an integrated clinical, molecular and serological classification of inflammatory bowel disease: Report of a Working Party of the 2005 Montreal World Congress of Gastroenterology. Can J Gastroenterol 2005; 19 (Supl. A): 5-36.

15. Alemayehu G, Jarnerot G. Colonoscopy during an attack of severe ulcerative colitis is a safe procedure and of great value in clinical decision making. Am J Gastroenterol 1991; 86: 187-90.

16. Carbonnel F, Lavergne A, Lemann M, et al. Colonoscopy of acute colitis: A safe and reliable tool for assessment of severity. Dig Dis Sci 1994; 39: 1550-7.

17. Horton K, Jones B, Fishman E. Imaging of the idiopathic inflammatory bowel diseases. In: Sartor R, Sandborn W, editors. Kirsner's inflammatory bowel diseases. $6^{\text {th }}$ ed. Philadelphia, PA: WB Saunders; 2004. p. 425-41.

18. Orel S, Rubesin S, Jones B, et al. Computed tomography vs. barium studies in the acutely symptomatic patient with Crohn's disease. J Comput Assist Tomogr 1987; 11: 1009-16.

19. Burton S, Liebig T, Frazier S, et al. High density oral barium sulfate in abdominal MRI: Efficacy and tolerance in a clinical setting. Magn Reson Imaging 1997; 15: 147-53.

20. Rollandi G, Martinolli C, Conzi R, et al. Magnetic resonance imaging of the small intestine and colon in Crohn's disease. Radiol Med 1996; $91: 81$.
21. Hata J, Haruma K, Suenaga K, et al. Ultrasonographic assessment of inflammatory bowel disease. Am J Gastroenterol 1992; 87: 4437.

22. Giaffer M, TindaleW, Holdworth D. Value of technetium-99m HMPAOlableled leukocyte scintigraphy as an initial screening test inpatients suspected of having inflammatory bowel disease. Eur J Gastroenterol Hepatol 1996; 8: 1995-2000.

23. Weldon M, Masoomi A, Britten A, et al. Quantification of inflammatory bowel disease activity using technetium-99m HMPAO labelled leucocyte single photon emission computerised tomography. Gut 1995; 36: 243-50

24. Darbari A, Sena L, Argani P, et al. Gadolinium-enhanced magnetic resonance imaging: A useful radiologic tool in diagnosing pediatric IBD. Inflamm Bowel Dis 2004; 10: 67-72.

25. Pio B, Byrne F, Aranda R, et al. Noninvasive quantification of bowel inflammation through positron emission tomography imaging of 2deoxy-2(18F)fluoro-D-glucose-labled white blood cells. Mol Imaging Biol 2003; 5: 271-7.

26. Pradel JA, David XR, Taourel P, et al. Sonographic assessment of the normal and abnormal bowel wall in nondiverticular ileitis and colitis. Abdom Imaging 1997; 22: 167-72.

27. Faure C, Belarbi N, Mougenot JF, et al. Ultrasonographic assessment of inflammatory bowel disease in children: Comparison with ileocolonoscopy. J Pediatr 1997; 130: 147-51.

28. Hollerbach S, Geissler A, Schiegl H, et al. The accuracy of abdominal ultrasound in the assessment of bowel disorders. Scand J Gastroenterol 1998; 33: 1201-8

29. Reimund JM, Jung-Chaigneau E, Chamouard P, et al. Diagnostic value of high resolution sonography in Crohn's disease and ulcerative colitis. Gastroenterol Clin Biol 1999; 23: 740-6.

30. Haber HP, Busch A, Ziebach R, et al. Ultrasonographic findings correspond to clinical, endoscopic, and histologic findings in inflammatory bowel disease and other enterocolitides. J Ultrasound Med 2002; 21: 375-82.

31. Albertini JL, Badran A, Freneaux E, et al. Technetium-99m HMPAO labelled leukocyte imaging compared with endoscopy, ultrasonography, and contrast radiology in children with inflammatory bowel disease. J Pediatr Gastroenterol Nutr 2001; 32: 278-86.

32. Limberg B, Osswald B. Diagnosis and differential diagnosis of ulcerative colitis and Crohn's disease by hydrocolonic sonography. Am J Gastroenterol 1994; 89: 1051-7.

33. Bru C, Sans M, Defelitto MM, et al. Hydrocolonic sonography for evaluating inflammatory bowel disease. AJR Am J Roentgenol 2001; 177: 99-105.

34. Pascu M, Roznowski AB, Muller HP, Adler A, Wiedenmann B, Dignass AU. Clinical relevance of transabdominal ultrasonography and magnetic resonance imaging in patients with inflammatory bowel disease of the terminal ileum and large bowel. Inflamm Bowel Dis 2004; 10: 373-82.

35. Regueiro M, Loftus EV Jr, Steinhart AH, Cohen RD. Clinical guidelines for the medical management of left-sided ulcerative colitis and ulcerative proctitis: Summary statement. Inflamm Bowel Dis 2006; 12: 972-8. 